\title{
Sparse Recovery Diagnosis Method Applied to Hybrid Dynamical System: The Case of Three-Phase DC-AC Inverter for Wind Turbine
}

\author{
Wafa Torki', Jean-Pierre Barbot ${ }^{2,3}$, Malek Ghanes ${ }^{3}$, Lassaad Sbita1 \\ ${ }^{1}$ Processes, Energy, Environment and electric Systems Laboratory, LR34ES18, National Engineering School of Gabes, Gabes, Tunisia \\ ${ }^{2}$ QUARTZ, EA 7393, ENSEA, Cergy, France \\ ${ }^{3}$ LS2N, UMR 6004, CNRS, Ecole Centrale de Nantes, Nantes, France \\ Email: tourkiwafa91@yahoo.fr
}

How to cite this paper: Torki, W., Barbot, J.-P., Ghanes, M. and Sbita, L. (2020) Sparse Recovery Diagnosis Method Applied to Hybrid Dynamical System: The Case of Three-Phase DC-AC Inverter for Wind Turbine. Open Access Library Journal, 7: e6776.

https://doi.org/10.4236/oalib.1106776

Received: September 3, 2020

Accepted: September 27, 2020

Published: September 30, 2020

Copyright $\odot 2020$ by author(s) and Open Access Library Inc.

This work is licensed under the Creative Commons Attribution International License (CC BY 4.0).

http://creativecommons.org/licenses/by/4.0/

\begin{abstract}
Power electronic systems tend to be great contributors to faults in many applications especially wind turbines because they are exposed to harsh operation conditions in height. Thus, a great attention has been paid to fault diagnosis technologies. In this paper, the concept of an exact recovery under a sparse fault assumption is applied to the diagnosis of three-phase DC-AC power electronic inverter, this method is denoted as Sparse Recovery Diagnosis (SRD). This method has the advantage to reconstruct on-line a vector of numerous faults from a few system measurements and with finite-time convergence. In this paper, the concept of an exact recovery under a sparse fault assumption is applied to the diagnosis of three-phase DC-AC power electronic inverter, this method is denoted as Sparse Recovery Diagnosis (SRD). In order to apply the proposed method, it is first necessary to have a dynamical modeling without and with each considered fault. After that, roughly speaking, some assumptions (Sparsity, Restrictive Isometry Property) are necessary with respect to the influence of the fault on the measured signals, in order to apply an exact SRD method. The algorithm used in this paper is based on homogeneous observer. Moreover, in order to take into account the quality of the measured noisy signals, the homogeneity degree is variable. The paper ends by some simulation results on a case study which highlight the well founded of the proposed algorithm with respect to previous algorithms that did not consider that the measurement is noisy.
\end{abstract}

\section{Subject Areas}

Electric Engineering 


\section{Keywords}

Sparse Recovery Diagnostic, Hybrid Dynamical System, Left-Invertibility, Restricted Isometry Property

\section{Introduction}

Reliability and availability of power electronics system are major concerns in many applications; automotive, industrial automation, converters for voltage and power control and green energy as wind energy conversion system (WECS) ... This later has paid considerable attention to fault diagnosis and maintenance because it is exposed to harsh operation conditions in height (e.g., [1] and [2]).

Thus, fault detection and isolation (FDI) has taken a considerable attention in power electronics applications, e.g., active filters, inverters for variable speed drive, etc... More and more, power electronics tend to be a great contributor to faults in modern wind turbines (e.g., [3] and [4]).

The FDI techniques in power electronics can be widely classified into three classes; 1 ) model-based in which the knowledge of system model is required including the faults effect (e.g., [5] [6] and [7]); 2) signal processing-based in which the spectral analysis is used to identify fault signatures (e.g., [8] and [9]) and 3) artificial intelligence-based that uses fuzzy logic and neural networks [10].

In our work, we focus on the model-based class where a new method based on Sparse faults assumption is proposed. This method amounts to left-invertibility problem which is treated in many works (e.g., in case of linear system in [11] and [12] and in case of non-linear in [13]. This method, denoted as Sparse Recovery Diagnosis (SRD), consists of recovering the information of faults (unknown inputs) from the system measurements (system outputs). This method is efficient when the number of unknown inputs is greater than the number of measurements.

In recent works (e.g., [14] and [15]), the (SR) algorithm is improved. Besides, it is applied in sensor networks [16], image processing and wireless communication [17], cyber-attacks [18] and mechanical faults (e.g., [19] and [20]).

The SRD technique requires a dynamic modeling in case of healthy and faulty operations. Hence, in this paper, a sparse representation of possible faults will be modeled and reconstructed on line. The considered faults are a variation of the DC-link voltage and a change in the filter output inductance value for each phase $\mathrm{a}, \mathrm{b}$ and $\mathrm{c}$.

Some assumptions (Sparsity, Restricted Isometry Property (RIP)) are needed with respect to the influence of faults on the measured signal, in order to apply an exact SR technique.

Therefore, the first contribution of this paper is the application of the SRD algorithm to detect and estimate electrical faults in hybrid dynamical system which has not been published yet. The second contribution consists on applying 
a finite time algorithm with an adaptive exponent $\alpha$ (applied in [21] as a fixed exponent) which takes into account the quality of the measured noisy signal.

The remainder of the paper is structured as follows; healthy and faulty models of the studied system are detailed in section II. Section III presents some recalls on left invertibility and SR algorithm as well as the presentation of the proposed FDI method; followed by comments and analysis simulation results in section IV. Concluding remarks end the paper.

\section{Modeling Framework}

\subsection{Healthy System Model}

The structure of the studied system is given in Figure 1. It is composed by a wind turbine connected to the grid under three-phase power electronics inverter, A DC-link and a RL filter (with R small as possible).

In this paper, we focused on the grid connected side which is modeled in $(a, b, c)$ reference frame by the following electrical equations [22]:

$$
\left\{\begin{array}{l}
v_{a n}=R_{a} i_{g a}+L_{a} \frac{\mathrm{d} i_{g a}}{\mathrm{~d} t}+v_{g a} \\
v_{b n}=R_{b} i_{g b}+L_{b} \frac{\mathrm{d} i_{g b}}{\mathrm{~d} t}+v_{g b} \\
v_{c n}=R_{c} i_{g c}+L_{c} \frac{\mathrm{d} i_{g c}}{\mathrm{~d} t}+v_{g c}
\end{array}\right.
$$

where, $v_{a n}, v_{b n}$ and $v_{c n}$ are the inverter output voltages, $i_{g a}, i_{g b}$ and $i_{g c}$ are the line currents, $v_{g a}, v_{g b}$ and $v_{g c}$ are the grid voltages and $L_{a, b, c}$ and $R_{a, b, c}$ are respectively the inductance and the resistance of the filter.

For the considered system, there are six switches that result 64 possible combinations. However, exactly eight operating modes are allowed because there is only one switch closed at any given time on each phase. Let the function $\sigma:[0, \infty) \rightarrow\{1,2, \cdots, 8\}$ denotes the active mode at time $t$, and let $s_{i}$, $i=1,2, \cdots, 6$, be an indicator variable that is equal to 0 whenever the switch $T_{i}$, $i=1,2, \cdots, 6$ is open and equal to 1 whenever it is closed. The switches of the same phase are controlled to be in complementary states. Then, Table 1 presents the possible open/closed switch positions, where, $s_{i}=1\left(\overline{s_{i}}=1-s_{i}=0\right)$ if $T_{i}$ is closed and $s_{i}=0\left(\bar{s}_{i}=1-s_{i}=1\right)$ if $T_{i}$ is open.

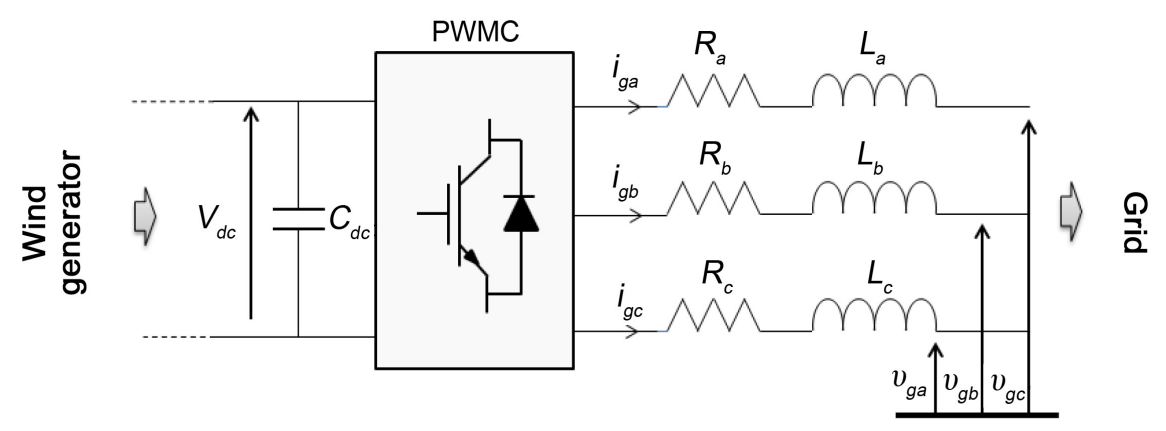

Figure 1. Structure of the grid side WECS. 
Table 1. Possible switch positions.

\begin{tabular}{lllllllll}
\hline & $\mathbf{1}$ & $\mathbf{2}$ & $\mathbf{3}$ & $\mathbf{4}$ & $\mathbf{5}$ & $\mathbf{6}$ & $\mathbf{7}$ & $\mathbf{8}$ \\
\hline $\mathrm{s}_{1} / \bar{s}_{2}$ & 0 & 0 & 0 & 0 & 1 & 1 & 1 & 1 \\
$S_{3} / \bar{s}_{4}$ & 0 & 0 & 1 & 1 & 0 & 0 & 1 & 1 \\
$S_{5} / \bar{s}_{6}$ & 0 & 1 & 0 & 1 & 0 & 1 & 0 & 1 \\
\hline
\end{tabular}

Considering ideal power electronic switches, the relation between the inverter output voltages and the DC-link voltage $V_{d c}$ is given by:

$$
\left\{\begin{array}{l}
v_{a n}-v_{b n}=\frac{V_{d c}}{2}\left(\left(s_{1}-s_{2}\right)-\left(s_{3}-s_{4}\right)\right) \\
v_{b n}-v_{c n}=\frac{V_{d c}}{2}\left(\left(s_{3}-s_{4}\right)-\left(s_{5}-s_{6}\right)\right) \\
v_{c n}-v_{a n}=\frac{V_{d c}}{2}\left(\left(s_{5}-s_{6}\right)-\left(s_{1}-s_{2}\right)\right)
\end{array}\right.
$$

Therefore, formalizing the above relations "Equation (1)" and "Equation (2)", the dynamics of the system can be described by the linear switched model "Equation (3)".

$$
M \frac{\mathrm{d} x(t)}{\mathrm{d} t}=R x(t)+P_{\sigma(t)} v(t)
$$

With

$$
\begin{gathered}
x(t)=\left[\begin{array}{lll}
i_{g a} & i_{g b} & i_{g c}
\end{array}\right]^{t}, \\
v(t)=\left[\begin{array}{llll}
v_{d c} & v_{g a} & v_{g b} & v_{g c}
\end{array}\right]^{t}, \\
M=\left[\begin{array}{ccc}
L_{a} & -L_{b} & 0 \\
0 & L_{b} & -L_{c} \\
1 & 1 & 1
\end{array}\right], \\
R=\left[\begin{array}{ccc}
-R_{a} & R_{b} & 0 \\
0 & -R_{b} & R_{c} \\
0 & 0 & 0
\end{array}\right]
\end{gathered}
$$

and

$$
P_{\sigma(t)}=\left[\begin{array}{cccc}
\frac{\left(s_{1}-s_{2}\right)-\left(s_{3}-s_{4}\right)}{2} & -1 & 1 & 0 \\
\frac{\left(s_{3}-s_{4}\right)-\left(s_{5}-s_{6}\right)}{2} & 0 & -1 & 1 \\
0 & 0 & 0 & 0
\end{array}\right]
$$

Here, the three phases are assumed to be identical (balanced three-phase system), i.e., $R_{a}=R_{b}=R_{c}=R$ and $L_{a}=L_{b}=L_{c}=L$. Then, by multiplying the both sides by $M^{-1}$ in (\ref\{eq3\}), we obtain a state-space model with the form: 


$$
\begin{aligned}
& \dot{x}(t)=A x(t)+B_{\sigma(t)} v(t) \\
& y(t)=C x(t)
\end{aligned}
$$

where:

$$
\begin{aligned}
& A=\left[\begin{array}{ccc}
-\frac{R}{L} & 0 & 0 \\
0 & -\frac{R}{L} & 0 \\
0 & 0 & -\frac{R}{L}
\end{array}\right], \\
& B_{\sigma(t)}=\left[\begin{array}{cccc}
p_{1} & -\frac{2}{3 L} & \frac{1}{3 L} & \frac{1}{3 L} \\
p_{2} & \frac{1}{3 L} & -\frac{2}{3 L} & \frac{1}{3 L} \\
p_{3} & \frac{1}{3 L} & \frac{1}{3 L} & -\frac{2}{3 L}
\end{array}\right], \\
& p_{1}=\frac{2\left(s_{1}-s_{2}\right)-\left(s_{3}-s_{4}\right)-\left(s_{5}-s_{6}\right)}{6 L} \text {, } \\
& p_{2}=\frac{-\left(s_{1}-s_{2}\right)+2\left(s_{3}-s_{4}\right)-\left(s_{5}-s_{6}\right)}{6 L} \\
& p_{3}=\frac{-\left(s_{1}-s_{2}\right)-\left(s_{3}-s_{4}\right)+2\left(s_{5}-s_{6}\right)}{6 L}
\end{aligned}
$$

and

$$
C=\left[\begin{array}{lll}
1 & 0 & 0 \\
0 & 1 & 0 \\
0 & 0 & 1
\end{array}\right]
$$

\subsection{Faulty System Model}

Several faults can affect the grid side wind energy conversion chain. Among them those refer to a continuous variation in certain parameters over the time which are occurred due to components degradation. For the switched system presented above, some faults are assumed to mainly happen. These main faults (one fault occurs at the same time) are the variation in output filter phases inductance and the variation in the DC-link voltage.

\section{Change in the DC-link voltage}

Consider the system of Figure 1, we assume the occurrence of a fault in the DC-link that could be an increase or decrease in the controlled DC-link voltage $v_{d c}$. This fault can be modeled by $v_{d c^{\prime}}=v_{d c}+\Delta v_{d c}$ where $v_{d c}$ represents the nominal DC-link voltage value and $\Delta v_{d c}$ describes the unknown fault magnitude. Thus, this fault causes a disturbance in the state space dynamics.

Without loss of generality, it is possible to write the system "Equation (4)" in two parts; one represents the healthy dynamics of the system and the other one 
captures the effect of the DC-link fault. This is shown in the following representation "Equation (5)".

$$
\dot{x}(t)=A x(t)+B_{\sigma(t)} v(t)+\phi_{1}(t) F_{1}
$$

where, $\phi_{1}(t)=\left[\begin{array}{lll}p_{1} & p_{2} & p_{3}\end{array}\right]^{t}$ and $F_{1}=\Delta V_{d c}$.

\section{Change in the output filter phase inductance}

This fault is assumed due to a variation of one inductance (i.e., $L_{i}=L+\Delta L_{i}$; $i \in\{a, b, c\})$ where $\mathrm{L}$ is the healthy inductance. For this type of fault we need to manipulate terms of the form $\frac{\mathrm{d}}{\mathrm{d} t}\left(L+\Delta L_{a}\right)$ in "Equation (1)" for the phase a. Assuming a constant inductance variation, this leads to a change in the matrix $M$ in "Equation (3)" that becomes

$$
\tilde{M}=\left[\begin{array}{ccc}
L+\Delta L_{a} & -L & 0 \\
0 & L & -L \\
1 & 1 & 1
\end{array}\right]
$$

This matrix is invertible, thus, the system dynamics given by (4) can be rewritten as follow:

$$
\dot{x}(t)=A x(t)+B_{\sigma(t)} v(t)+\phi_{2}(t) F_{2}
$$

where, $\phi_{2}(t)=\left[\begin{array}{llll}-2 K_{1} & K_{1} & K_{1}\end{array}\right]^{t}$ and $F_{2}=\frac{\Delta L_{a}}{3 L\left(3 L+2 \Delta L_{a}\right)}$,

With, $K_{1}=-3 R i_{g a}-3 v_{a}+\left(2 p_{11}+p_{22}\right) v_{d c} ; \quad p_{11}=\frac{\left(s_{1}-s_{2}\right)-\left(s_{3}-s_{4}\right)}{2}$ and $p_{22}=\frac{\left(s_{3}-s_{4}\right)-\left(s_{5}-s_{6}\right)}{2}$.

The same procedure will be followed for fault of the same type in phase b or $\mathrm{c}$. Thus, for the four considered faults with measurement noise, we have:

$$
\begin{gathered}
\dot{x}(t)=A x(t)+B_{\sigma(t)} v(t)+\sum_{i=1}^{4} \phi_{i} F_{i} \\
Y(t)=C x(t)+N(t)
\end{gathered}
$$

where, $\phi_{3}(t) F_{3}$ and $\phi_{4}(t) F_{4}$ represent respectively the quantities related to the change of the inductance value in phase $b$ and $c$ and they are described by:

$$
\begin{aligned}
& \phi_{3}(t)=\left[\begin{array}{lll}
K_{2} & -2 K_{2} & K_{2}
\end{array}\right]^{t} \text { and } F_{3}=\frac{\Delta L_{b}}{3 L\left(3 L+2 \Delta L_{b}\right)} ; \\
& \phi_{4}(t)=\left[\begin{array}{lll}
K_{3} & K_{3} & -2 K_{3}
\end{array}\right]^{t} \text { and } F_{4}=\frac{\Delta L_{c}}{3 L\left(3 L+2 \Delta L_{c}\right)},
\end{aligned}
$$

With,

$$
\begin{aligned}
& \qquad \begin{array}{l}
K_{2}=-3 R i_{g b}-3 v_{b}+\left(p_{22}-p_{11}\right) v_{d c} ; \backslash \\
K_{3}=-3 R i_{g c}-3 v_{c}-\left(p_{11}+p_{22}\right) v_{d c} \cdot M
\end{array} \\
& N(t)=\left[n_{1}(t), n_{2}(t), n_{3}(t)\right]^{\mathrm{T}} \text {, where } n_{i}(t), \quad i=1,2,3 \text {, are the uncorrelated } \\
& \text { white noises. }
\end{aligned}
$$


Due to the fact that all the relative degree with respect to faults $F_{i}$ is equal to one, the following algebraic equation is obtained

$$
\begin{aligned}
\bar{Y} & =\hat{\dot{Y}}-A x(t)-B_{\sigma(t)} v(t) \\
& =\Phi F+E(N, \delta)
\end{aligned}
$$

where $\Phi=\left[\phi_{1}, \phi_{2}, \phi_{3}, \phi_{4}\right] \in \mathfrak{R}^{3 \times 4}$ and $F=\left[F_{1}, F_{2}, F_{3}, F_{4}\right]^{\mathrm{T}}$. The vector $E(N, \delta)$ represents the error due to the measurement noise $(N)$ and numerical approximations $(\delta)$ (quantification, discretization ...).

$\hat{\dot{Y}}$ refers to the estimation of the derivative of $Y$ (measurement with noise) and it is given by the following differentiator [23]:

$$
\left\{\begin{array}{l}
\dot{\hat{Y}}=Z+\kappa_{1} \mu|Y-\hat{Y}|^{\theta} \operatorname{sign}(Y-\hat{Y}) \\
\ddot{\hat{Z}}=\kappa_{2} \mu^{2}|Y-\hat{Y}|^{2 \theta-1} \operatorname{sign}(Y-\hat{Y})
\end{array}\right.
$$

where $Z=\hat{\dot{Y}}+E(N, \delta)$ is the estimate of the derivative of $Y$, $|Y-\hat{Y}|^{\theta}=\left[\left|y_{1}-\hat{y}_{1}\right|^{\theta},\left|y_{2}-\hat{y}_{2}\right|^{\theta},\left|y_{3}-\hat{y}_{3}\right|^{\theta}\right]^{\mathrm{T}}$, $\operatorname{sign}(Y-\hat{Y})=\left[\operatorname{sign}\left(y_{1}-\hat{y}_{1}\right), \operatorname{sign}\left(y_{2}-\hat{y}_{2}\right), \operatorname{sign}\left(y_{3}-\hat{y}_{3}\right)\right]^{\mathrm{T}}, \quad \kappa_{1} \quad$ and $\kappa_{2}$ are positive constants and $\mu$ is the parameter in order to reject the perturbation.

From (10), setting

$$
\bar{Y}=Z-A x(t)-B_{\sigma(t)} v(t)
$$

Equation (9) is obtained.

\section{Faults Detection, Isolation and Estimations}

In this section, the main objectives are to detect, isolate and estimate the faults $F$ in (9). Note that in "Equation (9)", there are more unknown faults than "Equation (3)". However, in such considered filters, generally only one fault occurs at the same time. This assumption is necessary for faults estimation but not for faults detection. To achieve the objectives, the following criterion (12) is used [24]

$$
F^{*}=\arg \min \left\{\frac{1}{2}\|\bar{Y}-\Phi F\|_{2}^{2}+\lambda\|F\|_{0}\right\}
$$

where $\|F\|_{0}$ is the pseudo norm-zero (the number of non-zero elements).

Roughly speaking, the constraint $\lambda\|F\|_{0}$ allows to obtain a solution of (12) with a maximum of zero elements in $F$. Moreover, $\lambda$ is used as a threshold in order to avoid false alarms.

Unfortunately, (12) is difficult to solve with high dimension of $F$ containing many non-zero elements. In this case, in order to have an exact recovery of $F$, two assumptions are necessary. The first one is linked to verify that the number of equations is greater than two-times of non-zero elements of $F$ plus one. In our considered case, the non-zero elements of $F$ is one and the number of equations is three according to (9). Thus this first assumption is verified. The second one is the well-known Restricted Isometry Property (RIP) [25] for the matrix $\Phi$ 


$$
1-\gamma_{q} \leq e i g\left(\phi_{i}^{\mathrm{T}} \phi_{i}\right) \leq 1+\gamma_{q}
$$

where $\left.\gamma_{q} \in\right] 0,1[$ and each sub-matrix of dimension $3 \times 3$ of $\Phi$ must be regular.

In our considered case, this assumption is numerically and generically verified. Obviously, without current and voltage, the faults cannot be detected, isolated and estimated. Under these assumptions, optimization problem (12) is equivalent to the following optimization problem [25]

$$
F^{*}=\arg \min \left\{\frac{1}{2}\|\bar{Y}-\Phi F\|_{2}^{2}+\lambda\|F\|_{1}\right\}
$$

where, $\|F\|_{1}=\sum_{i}\left|F_{i}\right|$.

Then, a solution of the optimization problem of (14), providing that the matrix $\Phi$ satisfies the RIP condition (13), is given by the following algorithm (see [24] for linear version $\alpha=1$, [26] for homogeneous version $\alpha=] 0,1$ [ and [27] for homogeneous solution with variable exponent $\alpha$ ).

$$
\left\{\begin{array}{l}
\tau \dot{u}=-\left\lceil u(t)+\left(\Phi^{\mathrm{T}} \Phi-I_{4 \times 4}\right) \hat{F}-\Phi^{\mathrm{T}} \bar{Y}\right\rceil^{\alpha} \\
\hat{F}=a_{\lambda}(u) \\
\lceil\cdot\rceil \triangleq|\cdot|^{\alpha} \operatorname{sgn}(.)
\end{array}\right.
$$

where, $u \in \mathbf{R}^{4}$ represents the internal state vector, $\hat{F}$ refers to the estimated sparse signal $F, I_{4 \times 4}$ is the identity matrix in $\mathbf{R}^{4 \times 4}, \tau$ represents a constant that depends on the physical properties of the implementing system, $\alpha \in[0,1]$ is a variable exponential coefficient defined later and $a_{\lambda}(u)$ is a continuous soft thresholding function defined by:

$$
a_{\lambda}(u)= \begin{cases}0 & \text { if }|u| \leq \lambda \\ u-\lambda \operatorname{sgn}(u) & \text { if }|u|>\lambda\end{cases}
$$

The originalities of the paper are first to use the algorithm proposed in [21] in the context of diagnosis instead of the signal processing context (see (15)) secondly to adapt the $\alpha$ variable exponent method proposed in [27] at our problem. Doing so, the proposed fault detection algorithm is less sensible to noises (noise measurement, noises due to the differentiators' computation...). The proposed diagnosis algorithm with $\alpha$ variable is then given as

$$
\left\{\begin{array}{l}
\tau \dot{u}=-\left\lceil u(t)+\left(\Phi^{t} \Phi-I_{l}\right) \hat{F}-\Phi^{t} \bar{Y}\right]^{\alpha} \\
\hat{F}=a_{\lambda}(u) \\
\alpha=\frac{\zeta}{\zeta+\varepsilon} \\
\dot{\zeta}=-\tau \zeta+\tau\left|\bar{Y}_{h f}\right|
\end{array}\right.
$$

where the information, with respect to $E(N, \delta)$, is obtained by the following high past filter: 


$$
\left\{\begin{array}{l}
\bar{Y}_{h f}=\frac{s^{\prime 2}}{s^{\prime 2}+1.4142 s^{\prime}+1} \bar{Y} \\
s^{\prime}=\frac{s}{w_{c}}
\end{array}\right.
$$

where, $\bar{Y}_{h f}$ is the high frequency component of $\bar{Y}$ and for a well-chosen cutting frequency $w_{c}$, it is only function of $E(N, \delta)$. Note that in the systems (17) and (18), two filters are introduced. The first one (18) is a high pass second order filter (HF) which is the Butterworth Filter. The second one ( $\zeta$ dynamics) is a low pass first order filter (LF) with a nonlinear input that corresponds to the absolute value of $\bar{Y}_{h f}$.

Hence, the output of the (LF) generates the parameter $\alpha$ that is consequently variable with respect to the magnitude of the high frequency signal $\bar{Y}_{h f}$. The structure of the filters is implemented in Figure 2. Moreover, for $\alpha=0$, the system dynamic (17) becomes a first order sliding mode and the phenomenon of chattering occurs at equilibrium point but converges in finite time. For $\alpha=1$, the dynamic system (17) becomes the Locally Competitive Algorithm that is proposed in [24] and it converges exponentially to the equilibrium point.

Referring to the systems (17) and (18):

- $\varepsilon>0$ is a constant parameter which is chosen in the sequel to guarantee $\alpha$ in $[0,1[$. More accurately, when $\mathrm{Y}$ tends to 0 , i.e., noise-free signal (respectively tends to, i.e., very noisy signal) $\alpha$ tends to 0 , i.e., Levant differentiator(respectively tends to 1 , i.e., linear differentiator).

- $\tau$ is a positive constant parameter. It will be chosen sufficiently small in order to have a low frequency range.

- $w_{c}$ is the cut-off frequency of the HF.

- 14,142 is the damping ratio of HF that is obtained from the factors of the Butterworth Polynomial.

\section{Comparison with Some Others Existing Methods}

In the literature, it exists many diagnosis methods to resolve the considered problem. For example by hardware redundancy [28], if a sensor of DC voltage measurement is added, the problem becomes square and very easy to solve.

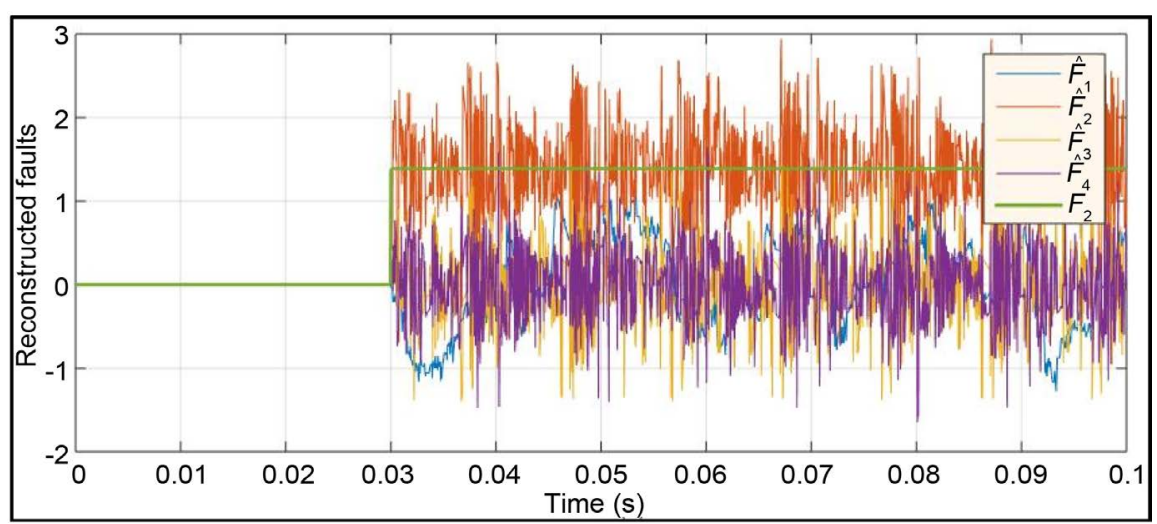

Figure 2. Deection of an inductance variation in the line phase a $F_{2}$ with $\alpha=0.5$. 
Another method based on a very well-known parity space analysis [28] can be used. In this case, one can define three residuals in order to detect and isolate the faults. The inputs of the residual are:

$$
\left\{\begin{array}{l}
\bar{r}_{1}=\bar{y}_{1}+2 \bar{y}_{2} \\
\bar{r}_{2}=\bar{y}_{2}+2 \bar{y}_{3} \\
\bar{r}_{3}=\bar{y}_{3}+2 \bar{y}_{1}
\end{array}\right.
$$

These residual inputs $\bar{r}_{i}$ are defined as linear combinations of $\bar{Y}$ components, in order to generate predefined residual input invariant with respect to predefined fault. More precisely the $\overline{r_{i}}$ are defined such that:

$$
\left\{\begin{array}{l}
\overline{r_{1}}=H_{1} \Phi F_{1}=0 \\
\overline{r_{2}}=H_{2} \Phi F_{2}=0 \\
\overline{r_{3}}=H_{3} \Phi F_{3}=0
\end{array}\right.
$$

With $H_{1}=[1,2,0], \quad H_{2}=[0,1,2], \quad H_{3}=[2,0,1]$.

And

$$
\Phi=\left(\begin{array}{cccc}
p_{1} & -2 K_{1} & K_{2} & K_{3} \\
p_{2} & K_{1} & -2 K_{2} & K_{3} \\
p_{3} & K_{1} & K_{2} & -2 K_{3}
\end{array}\right)
$$

As it is usual $\bar{r}_{i}, i=1,2,3$, are the inputs of a low pass filter, the outputs residual are denoted $r_{i}$. The alarm $a_{i}, i=1,2,3$ is activated $a_{i}=1$ when the absolute value of $r_{i}$ is greater than a threshold and off $a_{i}=0$ when the absolute value of $r_{i}$ is smaller than the threshold. This threshold plays a role that is similar to the parameter $\lambda$ used in "Equation (16)". Now by applying the parity space method, one has the following signature Table 2.

This comparison highlights the fact that they exist lot of competitive diagnosis methods and the presented method is only one of them.

This method is more competitive for a huge number of considered faults and in the case where more than one simultaneous fault is considered.

For example, the case of power network under cyber-attacks [18] highlights perfectly the well founded of the method.

Here the purpose is only to show this method can be useful in the diagnosis of hybrid dynamical system particularly to power electronic system.

\section{Simulations Results}

The system model and the designed diagnosis algorithm are implemented in Matlab/Simulink. Using an Euler solver with a fixed step equal to $5 \times 10^{-6}$, simulation results are obtained and presented below. The operated system parameters are given in Table 3. While, the algorithm parameters are $\tau=0.1 \mathrm{~s}$ (it is chosen sufficiently small in order to have a low frequency range), $\lambda=10^{-2}$ (according to the maximum variation that is tolerated before considering that there is a fault), $\kappa_{1}=2$ and $\kappa_{2}=1$ (using the pole placement) and $\mu=10^{4}$ (it is chosen greater than the maximum considered disturbance). 
Table 2. Signature table of parity space method.

\begin{tabular}{cccc}
\hline & $a_{1}$ & $a_{2}$ & $a_{3}$ \\
\hline No fault & 0 & 0 & 0 \\
$F_{1}$ & 1 & 1 & 1 \\
$F_{2}$ & 0 & 1 & 1 \\
$F_{3}$ & 1 & 0 & 1 \\
$F_{4}$ & 1 & 1 & 0 \\
\hline
\end{tabular}

Table 3. System parameters.

\begin{tabular}{cc}
\hline DC-link nominal voltage $v_{d c}$ & $800 \mathrm{~V}$ \\
DC-link capacitor $C$ & $2500 \mu \mathrm{F}$ \\
Switching frequency & $3 \mathrm{kHz}$ \\
Output filter inductance $L$ & $0.02 \mathrm{H}$ \\
Output filter resistance $R$ & $0.012 \Omega$ \\
Grid voltage $V_{\text {eff }}$ & $220 \mathrm{~V}$ \\
\hline
\end{tabular}

The application of the framework introduced in section III is implemented by analyzing four fault scenarios, i.e., in case of DC-link voltage variation and phase filter inductance variation.

In general the voltage sensor is less affected by noise and perturbation than current sensors. Then, the line currents $i_{g a}, i_{g b}$ and $i_{g c}$ are assumed to be measured. To these currents a white noise $N(t)$ of power 0.01 and sample time equal to $5 \times 10^{-6}$ (the simulation fixed step), is added. Thus $Y$ used in "Equation (10)" is obtained.

It is considered that only one fault can occur. Thus, algorithms (15) and (17) can be applied to estimate the occurred fault. The estimated value of $\bar{Y}$ (11) that is needed by both algorithms is given by "Equation (10)" where $\theta$ is fixed to 0.75 .

At first, we consider a fault causes an inductance to change at $t=0.03 \mathrm{~s}$. Setting $\Delta L_{a}$ equal to $30 \%$ of the nominal inductance value $L$. Then, two simulation results are illustrated in Figure 2 and Figure 3, respectively, in cases when the algorithm in (15) (exponent $\alpha$ is fixed to 0.5) and when the algorithm in (17) (the exponent $\alpha$ is variable) is used. The results show that the estimated fault is recovered and converged in finite time to its value. The non-zero estimated fault $\hat{F}_{2}$ related to the phase an inductance fault, converges to its corresponding value $\left(\frac{\Delta L_{a}}{3 L\left(3 L+2 \Delta L_{a}\right)}\right)$ while others estimated zero faults converged to zero in finite time. The difference between the estimated values in both cases is clearly shown; by using "Equation (17)" with $\alpha$ variable the results are much better than the case where "Equation (15)" is applied for $\alpha=0.5$.

Then, we assume the occurrence of a fault in the DC-link that causes a variation of $v_{d c}$ at $t=0.03 \mathrm{~s}$. The simulation results, given by Figure 4, show that the estimated non-zero fault $\hat{F}_{1}$ converges to its value $\left(\Delta v_{d c}=50 \mathrm{~V}\right)$ in finite time. The estimation of the recovered fault $\hat{F}_{1}$ in different cases of chosen exponent $\alpha$ (using "Equations (15) or (17)") is given by Figure 4. 
Taking the case when a fault occurs on the output filter inductance in the phase b (i.e., $F_{3}$ ) where $\Delta L_{a}=50 \% L$ at $t=0.3 \mathrm{~s}$, the simulation results are given by Figure 5 . The estimated fault $\hat{F}_{3}$ linked to the activated fault converges in finite time to its value. As it is clearly shown, the variable exponent $\alpha$ has a positive effect in fault estimation. In fact, the estimated fault for $\alpha$ variable (using "Equation (17)") is close to its desired value than the fault recovered using "Equation (15)" where $\alpha$ is fixed.

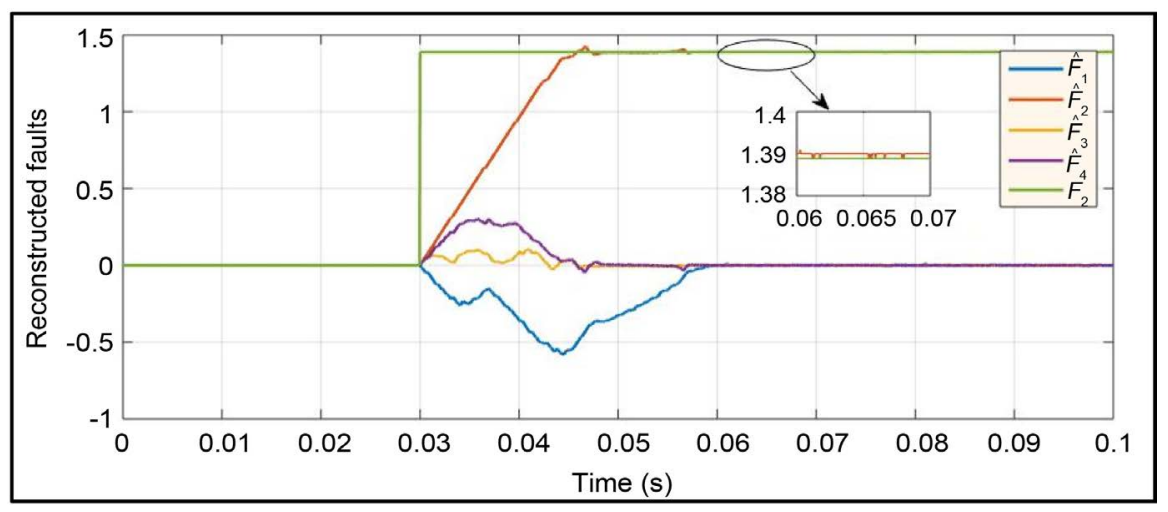

Figure 3. Detecion of an inductance variation in the line phase a $F_{2}$ with $\alpha$ variable.

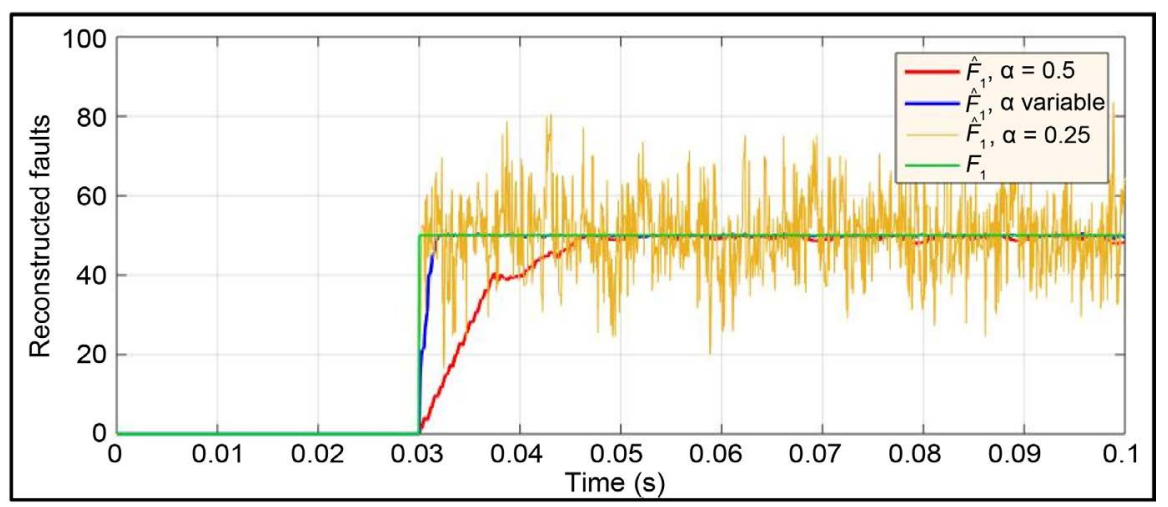

Figure 4. Detecion of a DC-link voltage variation $F_{1}$ with $\alpha=0.25, \alpha=0.5$ and $\alpha$ variable.

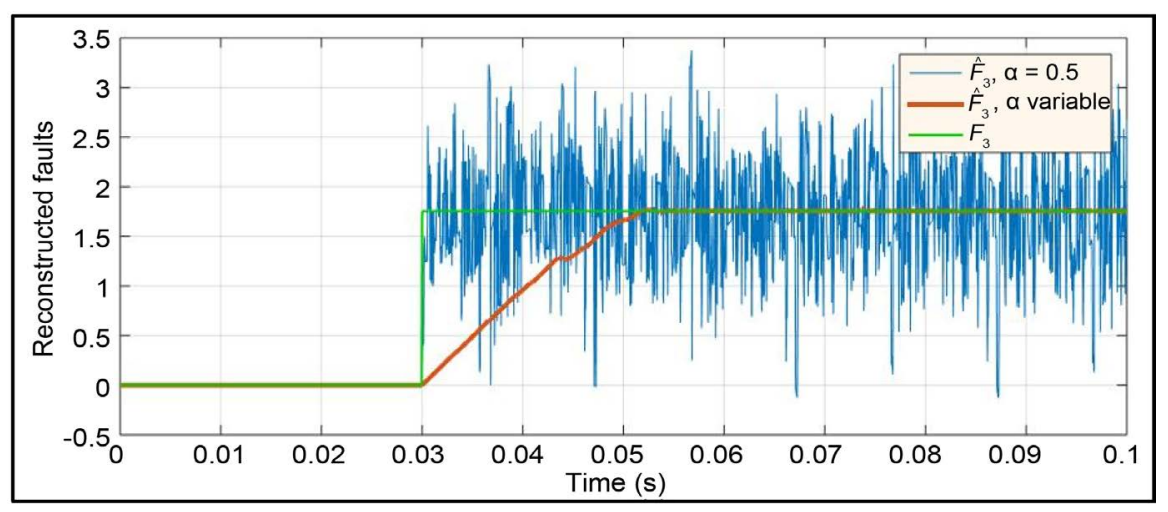

Figure 5. Detecion of an inductance variation in the phase $\mathrm{b} F_{3}$ with $\alpha$ variable and fixed to $\alpha=0.5$. 
The above results have proven that the algorithm "Equation (17)" with $\alpha$ variable performs well in presence of noise and then the performance of the proposed optimization system is improved.

\section{Conclusion}

In this work, healthy and faulty dynamical models of grid side WECS were presented and the results were carried out via Matlab/Simulink environment. A Sparse Recovery (SR) algorithm was applied to detect and estimate a fault affecting the output filter phases inductance and the DC-link voltage. The exponent of this algorithm $\alpha$ may be chosen between 0 and 1. When $\alpha=0$ this corresponds to a sliding mode solution and cancels the perturbation and when $\alpha=1$ this corresponds to linear solution less sensible to noise. Then, it was proposed a solution with $\alpha$ variable in order to take into account the noise evolution. Therefore, the dynamic of $\alpha$ was designed with respect to the high frequency magnitude of the signal. For the considered cases, the comparison of the simulation results highlights the well-founded of the SR algorithm with $\alpha$ variable. As a future work, we will focus on the stability analysis of the proposed SR algorithm with a varying exponent gain $\alpha$ and on an experimental validation of the proposed algorithm to recover faults in finite time.

\section{Conflicts of Interest}

The authors declare no conflicts of interest regarding the publication of this paper.

\section{References}

[1] Liu, W., Tang, B., Han, J., Lu, X., Hu, N., and He, Z. (2015) The Structure Healthy Condition Monitoring and Fault Diagnosis Methods in Wind Turbines: A Review. Renewable and Sustainable Energy Reviews, 44, 466-472. https://doi.org/10.1016/j.rser.2014.12.005

[2] Kamel, R.M. (2014) Effect of Wind Generation System Types on Micro-Grid (MG) Fault Performance during Both Standalone and Grid Connected Modes. Energy Conversion and Management, 79, 232-245. https://doi.org/10.1016/j.enconman.2013.12.009

[3] Spinato, F., Tavner, P.J., van Bussel, G.J.W. and Koutoulakos, E. (2009) Reliability of Wind Turbine Subassemblies. IET Renewable Power Generation, 3, 387-401. https://doi.org/10.1049/iet-rpg.2008.0060

[4] Yang, S., Bryant, A., Mawby, P., Xiang, D., Ran, L. and Tavner, P. (2011) An Industry-Based Survey of Reliability in Power Electronic Converters. IEEE Transactions on Industry Applications, 47, 1441-1451. https://doi.org/10.1109/TIA.2011.2124436

[5] Ding, S. (2008) Model-Based Fault Diagnosis Techniques: Design Schemes, Algorithms, and Tools. Springer-Verlag, London.

[6] Zhang, D., Wang, F., Burgos, R., Kern, J., El-Barbari, S. and Boroyevich, D. (2009) Internal Fault Detection and Isolation for Paralleled Voltage Source Converters. 2009 Twenty-Fourth Annual IEEE Applied Power Electronics Conference and EXposition, Washington DC, 15-19 February 2009, 833-839.

https://doi.org/10.1109/APEC.2009.4802758 
[7] de Araujo Ribeiro, R., Jacobina, C., da Silva, E. and Lima, A. (2003) Fault Detection of Open-Switch Damage in Voltage-Fed PWM Motor Drive Systems. IEEE Trans. Power Electron, 18, 587-593. https://doi.org/10.1109/TPEL.2003.809351

[8] Chowdhury, F. and Aravena, J. (1998) A Modular Methodology for Fast Fault Detection and Classification in Power Systems. IEEE Transactions on Control Systems Technology, 6, 623-634. https://doi.org/10.1109/87.709497

[9] Turpin, C., Baudesson, P., Richardeau, F., Forest, F. and Meynard, T. (2002) Fault Management of Multicell Converters. IEEE Transactions on Industrial Electronics, 49, 988-997. https://doi.org/10.1109/TIE.2002.803196

[10] Masrur, M.A., Chen, Z. and Murphey, Y. (2010) Intelligent Diagnosis of Open and Short Circuit Faults in Electric Drive Inverters for Real-Time Applications. IET Power Electronics, 3, 279-291. https://doi.org/10.1049/iet-pel.2008.0362

[11] Silverman, L. (1969) Inversion of Multivariable Linear Systems. IEEE Transactions on Automatic Control, 14, 270-276.

[12] Sain, M. and Massey, J. (1969) Invertibility of Linear Time-Invariant Dynamical Systems. IEEE Transactions on Automatic Control, 14, 141-149. https://doi.org/10.1109/TAC.1969.1099133

[13] Barbot, J.-P., Boutat, D. and Floquet, T. (2009) An Observation Algorithm for Nonlinear Systems with Unknown Inputs. Automatica, 45, 1970-1974. https://doi.org/10.1016/j.automatica.2009.04.009

[14] Sun, X. and Zhang, J. (2014) An Exact First-Order Algorithm for Decentralized Consensus Optimization.

[15] Elad, M., Figueiredo, M.A. and Ma, Y. (2010) On the Role of Sparse and Redundant Representations in Image Processing. Proceedings of the IEEE, 98, 972-982. https://doi.org/10.1109/JPROC.2009.2037655

[16] Wong, K.I., Vong, C.M., Wong, P.K. and Luo, J. (2015) Sparse Bayesian Extreme Learning Machine and Its Application to Biofuel Engine Performance Prediction. Neuro Computing, 149, 397-404. https://doi.org/10.1016/j.neucom.2013.09.074

[17] Majumdar, A., Ansari, N., Aggarwal, H. and Biyani, P. (2016) Impulse Denoising for Hyper-Spectral Images a Blind Compressed Sensing Approach. Signal Processing, 119, 136-141. https://doi.org/10.1016/j.sigpro.2015.07.019

[18] Nateghi, S., Shtessel, Y., Barbot, J.-P., Zheng, G. and Yu, L. (2018) Cyber-Attack Reconstruction via Sliding Mode Differentiation and Sparse Recovery Algorithm: Electrical Power Networks Application. 15th International Workshop on Variable Structure Systems ( VSS), Graz, Austria, 9-11 July 2018, 285-290. https://doi.org/10.1109/VSS.2018.8460426

[19] Wang, H., Ke, Y., Song, L., Tang, G. and Chen, P. (2016) A Sparsity-Promoted Decomposition for Compressed Fault Diagnosis of Roller Bearings. Sensors, 16, 1524. https://doi.org/10.3390/s16091524

[20] Huang, W., Sun, H. and Wang, W. (2017) Resonance-Based Sparse Signal Decomposition and Its Application in Mechanical Fault Diagnosis: A Review. Sensors, 17, 1279. https://doi.org/10.3390/s17061279

[21] Yu, L., Zheng, G. and Barbot, J.-P. (2017) Dynamical Sparse Recovery with Finite-Time Convergence. IEEE Transactions on Signal Processing, 65, 6146-6157. https://doi.org/10.1109/TSP.2017.2745468

[22] Ding, X., Poon, J., Čelanović, I. and Dominguez-Garcia, A.D. (2013) Fault Detection and Isolation Filters for Three-Phase AC-DC Power Electronics Systems. IEEE Transactions on Circuits and Systems I: Regular Papers, 60, 1038-1051. https://doi.org/10.1109/TCSI.2012.2221222 
[23] Levant, A. (2005) Homogeneity Approach to High-Order Sliding Mode Design. Automatica, 41, 823-830. https://doi.org/10.1016/j.automatica.2004.11.029

[24] Balavoine, A., Rozell, C.J. and Romberg, J. (2013) Convergence Speed of a Dynamical System for Sparse Recovery. IEEE Transactions on Signal Processing, 61, 4259-4269. https://doi.org/10.1109/TSP.2013.2271482

[25] Candès, E.J. and Wakin, M.B. (2008) An Introduction to Compressive Sampling. IEEE Signal Processing Magazine, 25, 21-30. https://doi.org/10.1109/MSP.2007.914731

[26] Balavoine, A., Romberg, J. and Rozell, C. (2013) Correction to Convergence and Rate Analysis of Neural Networks for Sparse Approximation. IEEE Transactions on Neural Networks and Learning Systems, 25, 1595-1596.

https://doi.org/10.1109/TNNLS.2013.2292700

[27] Ren, J., Yu, L., Lyu, C., et al. (2019) Dynamical Sparse Signal Recovery with Fixed-Time Convergence. Signal Processing, 162, 65-74.

https://doi.org/10.1016/j.sigpro.2019.04.010

[28] Ghanes, M., Barbot, J.P., Fridman, L. and Levant, A. (2017) A Novel Differentiator: A Compromise between Super Twisting and Linear Algorithms. 2017 IEEE 56th Annual Conference on Decision and Control (CDC), Melbourne, 12-15 December, 2017, 5415-5419. https://doi.org/10.1109/CDC.2017.8264460 